und der Trägheit infolge der Elektrisierung innerer Oberflächen innerhalb der Lösung $a b$ hängt. Nach dieser Anschauung würde die Abnahme und das schliebliche Verschwinden der Potentialdifferenz zwischen den Glutinteilchen und dem Wasser, sobald die Konzentration der Säure über einen bestimmten Wert steigt, auf die Unterdrückung der schwachen Ionisation des Salzes durch einen Säureüberschuß zurückzuführen sein.

\title{
Einige Bestätigungen meiner Hypothesen über die Koagulation.
}

Der berühmte italienische Physiker Prof. August Righi hat in seinem Rektorats-Vortrag „Die neueren Ansichten über die innere Struktur der Materie", den er in der italienischen Gesellschaft für den Fortschritt der Wissenschaften in Parma gehaiten hat, in einer bewundernswürdigen Synthese alle die Argumente zusammengestellt, welche in der letzten Zeit geltend gemacht worden sind und am besten geeignet erscheinen, die innere Struktur der Materie zu bestimmen. Er beschäftigt sich mit den mannig* fachen Theorien über die innere Struktur der Kolloide, und äußert sich speziell über die auch nach seiner Meinung elektrische Natur der kolloiden Lösungen, indem er alle die Tatsachen aufführt, welche die Annahme einer elektrischen Ladung in den Kolloiden notwendig machen. Er spricht in der bezeichneten Weise von der Koagulation und weist auf eine höchst wichtige Tatsache hin, durch welche man genötigt ist zuzugeben, daß die Koagulation von der Neutralisation von elektrischen Ladungen abhängt: "Wenn man eine Lösung eines negativen Kolloids (z. B. des Alkali-Globulins des Ochsenserums) der Strahlungswirkung eines Radiumsalzes aussetzt, so erhält man eine allmähliche (graduelle) Koagulation, während dies nicht geschieht, wenn man statt dessen in dem Versuch ein positives Kolloid anwendet, welches vielmehr manchmal noch flüssiger zu werden scheint. Da die $\beta$-Strahlen, welche die Flüssigkeit durchdringen, nur zum kleinsten Teile absorbiert werden, die $\boldsymbol{\alpha}$-Strahlen aber vollständig, so ist es natürlich, die Koagulation den elektrischen Ladungen zuzuschreiben, die das Kolloid von diesen letzteren empfangen hat. Läßt man dagegen auf ein positives Kolloid (z. B. Eisenhydroxyd) nicht mehr die $\alpha$-Strahlen, sondern nur die $\beta$-Strahlen einwirken, so erhält man ebenfalls, wenn auch langsamer, Koagulation." Ferner sagt er unter Hinweis auf die ebenso wichtige Tatsache der Koagulation durch Elektrolyte: „Es ist natürlich,

1) Siehe auch: Ber. der chem. Gesellsch. Roms, 12. April 1908. daß in diesem Falle die Erscheinung der Koagulation nunmehr auf die neutralisierende Ladung eines der Ionen zurückzuführen ist, was jedenfalls eine präponderierende Wirkung erklären würde." Diese letzten Worte des berühmten Physikers des Bologneser Atheneums gehen mich sehr nahe an, da von mir zuerst systematische und sehr in diesem Sinne übereinstimmende Experimente veröffentlicht wurden, und da die Hypothese, dab die Koagulation auf ein Phänomen der Diffusion und zugleich der Neutralisation von elektrischen Ladungen des einen der lonen zurückzuführen ist, von mir zuerst formuliert wurde.

Viele Autoren haben sich mit der Koagulation beschäftigt, aber meist waren die experimentellen Daten ohne die geringste Uebereinstimmung und die Experimente waren nicht systematisch an Gruppen von Substanzen ausgeführt, so wie man mit gesunder wissenschaftlicher Kritik die Natur der Koagulation und der kolloiden Lösungen hätte untersuchen sollen.

In der Sitzung vom 13. Dezember 1904 brachte ich in der chemischen Gesellschaft $z u$ Rom zur Kenntnis, dab die Koagulationswirkungen von den Elektrolytenherrühren und nicht von den in der Lösung nicht dissoziierten organischen Körpern - und dab in der Reihe der Alkalimetalle die Geschwindigkeit, mit welcherdiekoagulation der kolloiden Kieselsäure hervorgerufen wird, sich erhöht mit dem wachsenden Atomgewicht des Kations, so daB vom Lithium zum Cäsium fortschreiterd das Ammonium zwischen Natrium und Kaliumzu stehenkommt.

Diese experimentellen Resultate waren von solcher Wichtigkeit, dab sie die folgenden Worte des berühmten Prof. Giuseppe Bruni von der Universität Padua hervorriefen, der so tief in die physikalische Chemie eingedrungen ist: „Dieses höchst interessante Resultat ist geeignet, viel Licht auf die Theorie zu werfen, 
und es ist zu wünschen, daß Dr. Pappadà in seinen Untersuchungen fortfahren möge." Später, am 30. Mai 1906, brachte die „Gazz. chim. ital." andere interessante Resultate zur Kenntnis :

Die koagulierende Wirkung eines Elektrolyten hängt ab von der Diffusionsgeschwindigkeit und von der elektrischen Ladung des Kations und wächst mit deren $Z$ unahme, chemisch äquivalente Konzentrationen der Elektrolytevorausgesetzt.

Bei Kationen mit der gleichen Ladung hängt die Koagulationswirkung nurvonder Diffusionsgeschwindigkeit ab und wächst mit dieser.

Auf Grund dieser Resultate, die bei vielen und wichtigen Kolloiden übereinstimmen, hatte ich mir erlaubt, auf wissenschaftlicher Basis meine Hypothese über die Koagulation zu formulieren:

Die Lösung eines Elektrolyten, die mit der kolloiden Lösung in Berührung gebracht ist, wird sich in dieser ausbreiten, um die gleiche Konzentration an ihren verschiedenen Punkten zuerreichen. Die Elektrolyte werden nach der Dissoziationstheorie nicht als ganze Moleküle diffundieren können, sondern als freie lonen und mit eigener Geschwindigkeit; diefesten Teilchen der Kolloide, welche für sie nicht durchlässig sind, werden Stöbe empfangen, welche das Bestreben haben werden, sie ihrem $Z$ ustand feinster $S$ uspension $z$ u entreiben, und $z$ war in mehr oder weniger kurzer Zeit, entsprechend der Geschwindigkeit, mit der das lon diffundiert. Ionen, welche mit positiver Elektrizität geladen sind, werden die negative Ladung der Teilchen neutralisieren, während für die Kationen selbst sich dieursprüngliche Ladung wieder herstellen wird, da das positiv geladeneWasserdie eigene elektrische Ladung abgeben wird. Es ist natürlich, dab die kolloiden Teilchen nicht mehr in sichtlicher Lösung bleiben, sondern dab sie gefällt werden müssen: da ja ihre Suspension, wie gesagt, nur in dem Falle stattindet, dab sie eine negative Ladung haben; wie übrigens a uch die Existenz eines Ions in Lösung nurdann möglich ist, solange es elektrische Ladung hat.
In der Mitteilung von 1906 sagte ich auch, daB die Koagulation durch Elektrolyte unter dem Gesichtspunkt der Diffusion im Wasser und der elektrischen Ladung der lonen betrachtet werden mub. Kurz, das elektrisch geladene kolloide Teilchen ist nur dann imstande die eigene Ladung zu neutralisieren, wenn es Stöße von den diffundierenden lonen erhalten hat.

Bei der Koagulation muB man sich daher nicht nur den elektrischen Zustand der feinen Suspension des Kolloids vergegenwärtigen, sondern auch die Natur der kolloiden (suspendierten) Substanz, und zwar muB man nachsehen, ob diese durchlässig, halbdurchlässig oder undurchlässig ist. Die durchlässigen Kolloide bieten der freien Diffusion der Elektrolyte im Wasser kein Hindernis und dürften deswegen nach meiner Hypothese nicht durch die Gegenwart der gleichen Elektrolyten koaguliert werden: und dies ist in der Tat der Fall. Die Geschwindigkeit der Diffusion und die entsprechende Diffusibilität ändern sich nicht, wenn die Kristalloide verschiedene Kolloide durchdringen, wie Gelatine, Albumin, das Mucilagin von Tragantgummi, Stärkekleister usw. Ich habe sorgfältig $\mathrm{zu}$ erhärten gesucht, daß die Lösungen der genannten Kolloide nicht gefällt werden durch die Gegenwart der Elektrolyten, unter identischen Bedingungen, unter denen die Fällung der anderen Kolloide eintritt, wie z. B. die des Berliner Blau, des Eisenkupferzyanürs, der Kieselsäure, des kolloiden Silbers, Goldes und Platins; diese Kolloide sind nicht durchlässig und empfangen daher Stöße, deren Effekt ist, das kolloide Teilchen zur Neutralisation seiner eigenen Ladung zu befähigen.

In derselben Mitteilung von 1906 brachte ich in betreff des elektrischen Zustandes der Kolloide zur Kenntnis: Die Kolloide folgen, wie seit längerer Zeit bekannt ist, und wie ich binnen kurzem mitwichtigeren Einzelheiten bestätigen werde, in bezug aufden Strom dem Verhalten der echten Lösungen, mit Ausnahme jedoch der Metallhydroxyde, welche sich anders verhalten; und meine Experimente über die Koagulation durch Elektrolyte zeigen für das Eisenhydroxydeinen tiefgreifenden Unterschied gegen das einförmige Verhalten der übrigen bis jetzt von mir genau untersuchten Kolloide.

Ich möchtef $\in$ rner feststellen, daB für dieses Kolloid (immerzufolgeder Mitteilung von 1906) die Koagulations- 
experimente ganzanalogdenen sind, welche schon von dem berühmten Prof. Federigo Giolitti veröffentlicht worden sind. Die Säuren Chlorwasserstoff, Bromwasserstoff, Jodwasserst off, Salpetersäure, die Chlorüre, Bromüre, Jodüre, immer in Zehntel. normallösungen, koagulieren nicht, während die Schwefelsäure und die Sulfate in den gleichen Konzentrationen koagulierenund untersichmit variierendem Kation keinemerkliche Verschiedenheit in der koagulierenden Wirkung zeigen.

Dieses interessante Resultat der Koagulation des Eisenoxydhydrats stimmt überein mit einem andern, welches gestattet, das Eisenoxydhydrat als positives Kolloid anzusehen.

Wenn das Eisenhydroxyd ein positives Kolloid ist, so wird die Neutralisation der Ladung der kolloiden Teilchen mit Hilfe der negativen Ladung des Anions zustande kommen. Und dab dies in dieser Weise stattfindet, das zeigen auf das klarste meine Experimente, die in der Mitteilung von 1906 zusammengefaßt sind.

Zwischen den Säuren Chlor-, Brom-, Jodwasserstoff, den Bromüren, Jodüren, Chlorüren,
Nitraten habe ich keinen bemerkenswerten Unterschied in der Koagulationswirkung beobachtet, da die Anionen $\mathrm{Cl}^{\prime}, \mathrm{Br}^{\prime}, \mathrm{J}^{\prime}, \mathrm{NO}_{3}^{\prime}$ die gleiche Diffusionsgeschwindigkeit und gleiche elektrische Ladung haben.

Die Schwefelsäure indessen und die Sulfate koagulieren in Zehntelnormallösung das Eisenoxydhydrat, wobei bei ihnen dem Variieren des Kations keine bemerkenswerte Verschiedenheit in der koagulierenden Wirkung entspricht. Dies verhält sich darum so, weil die Neutralisation der positiven Ladung $\operatorname{des}^{\circ}$ Kolloids mittelst der negativen Ladung des Anions zustande kommen muß, und das $\mathrm{SO}_{4}{ }^{\prime}$ daher durch eine doppelte Ladung eine stärkere koagulierende Wirkun ghaben wird als $\mathrm{Cl}^{\prime}, \mathrm{Br}^{\prime}, \mathrm{J}^{\prime}, \mathrm{NO}_{3}^{*}$. Folglich wird das Kation keine bedeutende Rolle bei diesen Koagulationsvorgängen spielen.

Ich schlieBe aus alledem, daß meine Hypothese über die Koagulation meine zahlreichen Experimente zu einer harmonischen Einheit verbindet, die ultramikroskopischen Beobachtungen, den elektrischen Transport durch den Strom und die Koagulation durch die Ausstrahlungen von Radiumsalzen, und zwar sowohi bei Einwirkung der $\alpha$-Strahlen oder der $\beta$-Strahlen.

\title{
Die Untersuchung von Stas über Chlorsilberlöslichkeit.
}

\author{
Von Karl Dracker.
}

Die kürzích in dieser Zeitschrift' ${ }^{1}$ ) mitgeteilten Studien Svedberg's erinnern an eine große Untersuchung, die Stas als Hilfsarbeit $z u$ seinen Atomgewichtsbestimmungen im Jahre 1872 ausgeführt hat. Sie führt den Titel "Recherches de statique chimique au sujet du chlorure et du bromure d'argent " ${ }^{2}$ ) und enthălt auker vielen anderen interessanten Ergebnissen folgende für die Kolloidlehre bedeutungsvolle Mitteilungen.

Stas unterscheidet folgende Formen des Chlorsilbers:

1. gelatineux - 2. caseeux, floconneux 3. pulvérulent - -4 . grenu, écailleux, cristallin, fondu. -

Diese vier Gruppen zeigen sehr starke Verschiedenheit im Verhalten gegen Wasser und Salzlösungen sowohl wie gegen Licht, dergestalt, daß die letzte Gruppe überhaupt nicht

1) Koll.-Zeitschr. 4, 49 (1909).

2) Oeuvr. compl. 1, 87 . nachweisbar löslich ist (soweit die Methoden der damaligen Zeit reichen), während die beiden mittleren Gruppen merkliche Löslichkeit zeigen, und die erste, die Stas nicht untersucht hat, so stark dispergiert ist, dab äie Bestimmung der Löslichkeit auf die schwer zu losende Vorfrage der Unterscheidung zwischen gelöstem und emulgiertem resp. suspendiertem Stoffe stöbt.

Was das Verhalten gegen Licht betrifft, so zeigen Stas' Angaben in Ueboreinstimmung mit heute wohlbekannten Erfahrungers, dab die gleiche Reihenfolge besteht wie bezüglich der Löslichkeit, ansteıgend von der vierten bis zur zweiten Gruppe. Stas bemerkt ousdrücklich, daB von allen Zuständen des Chlorsilbers der käsige, flockige die höchste Lichtempfindlichkeit zeige.

Für die Darstellung der verschiedenen Formen gibt St a s ausführliche Vorschriften. Sie können hier nicht im vollen Lmfange mitgeteilt werden; es sei nur auf eine besonders interessante Beobachtung hingewiesen. St as schreibt über 